\title{
Use of ICDAS Combined with Quantitative Light-Induced Fluorescence as a Caries Detection Method
}

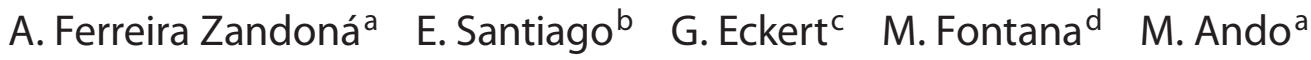 \\ D.T. Zero ${ }^{a}$ \\ a Department of Preventive and Community Dentistry, Indiana University School of Dentistry, and \\ ${ }^{b}$ Department of Medicine, Indiana University School of Medicine, Indianapolis, Ind.; ${ }^{\mathrm{C}}$ Department of \\ Cariology and Restorative Sciences, University of Michigan School of Dentistry, Ann Arbor, Mich.; \\ ${ }^{\mathrm{d}}$ Research Center, University of Puerto Rico School of Dentistry, San Juan, P.R., USA
}

\section{Key Words}

Dental caries • International Caries Detection and

Assessment System • Longitudinal study • Quantitative

light-induced fluorescence

\begin{abstract}
The purpose of this study was to combine a standardized visually based system, the International Caries Detection and Assessment System (ICDAS), with a sensitive fluorescence-based system, quantitative light-induced fluorescence (QLF), to determine the ability to monitor caries lesion progression. This combination (QLF-I) has the potential to increase the sensitivity of the visual method without compromising specificity. A total of 460 children were enrolled and examined at baseline, 8 months and 12 months by ICDAS and QLF by a single examiner. The examiner repeatability for both methods was comparable, varying between weighted kappa of 0.70 and 0.79 . The DMFT score was 6.0 (SD 5.8) at baseline and 6.4 (SD 6.3) at 12 months, and both methods were able to follow the increase in incidence. The QLF-I scored more surfaces at the early ICDAS scores ( 1 and 2 ) and score 4 . Not all lesions progressed at the same rate, differing by score at baseline and surface type.
\end{abstract}

Copyright $\odot 2010$ S. Karger AG, Basel
() 2010 S. Karger AG, Basel

Fax +41613061234 E-Mail karger@karger.ch www.karger.com
Accessible online at: www.karger.com/cre
The International Caries Detection and Assessment System (ICDAS) was developed as a standardized system based on evidence that should lead to better quality information to enlighten decisions about the appropriate diagnosis, prognosis and clinical management of dental caries at both the individual and public health levels [Pitts, 2004]. The ICDAS has been shown to be reproducible and accurate [Jablonski-Momeni et al., 2008] and to allow the detection and assessment of early lesions and longitudinal follow-up [Burt et al., 2006; Ekstrand et al., 2007; Finlayson et al., 2007; Ismail et al., 2007, 2008; Sohn et al., 2007; Cook et al., 2008; Jablonski-Momeni et al., 2008; Varma et al., 2008]. As originally conceived, the ICDAS is a 'wardrobe' of validated tools that should allow users to select the best criteria and conventions for a specific use [Pitts, 2004]. To date, no studies have reported on how this 'wardrobe' system can be used with some of the technology-based caries detection methods currently available. The combination of the ICDAS with a technology-based method has the potential to bring forward the best characteristics of each method.

One of the nonconventional detection methods that has received great attention is quantitative light-induced fluorescence (QLF). This method has been shown to be sensitive for the detection of early incipient lesions [de Jos- 
Table 1. ICDAS and QLF-I criteria

\begin{tabular}{|c|c|c|}
\hline & ICDAS criteria & QLF-I criteria (modified ICDAS) \\
\hline 0 & Sound tooth surface & Sound tooth surface \\
\hline 1 & First visual change in enamel & Slight fluorescence change \\
\hline 2 & Distinct visual change in enamel & Distinct fluorescence change \\
\hline 3 & $\begin{array}{l}\text { Localized enamel breakdown due to caries with no visible } \\
\text { dentin }\end{array}$ & $\begin{array}{l}\text { Visible enamel breakdown with a distinct fluorescence } \\
\text { change }\end{array}$ \\
\hline 4 & $\begin{array}{l}\text { Surface with underlying dark shadow from dentin with or } \\
\text { without enamel breakdown }\end{array}$ & $\begin{array}{l}\text { Poorly delineated distinct fluorescence change with or } \\
\text { without enamel breakdown }\end{array}$ \\
\hline 5 & Distinct cavity with visible dentin & $\begin{array}{l}\text { Cavitation visible with distinct fluorescence change } \\
\text { (5 and 6) }\end{array}$ \\
\hline 6 & Extensive distinct cavity with visible dentin & Collapsed with 5 \\
\hline
\end{tabular}

selin de Jong et al., 1995; Ando et al., 1997, 2001, 2004b; Angmar-Mansson and ten Bosch, 2001; Shi et al., 2001; Tranaeus et al., 2001; Amaechi et al., 2003; Gonzalez-Cabezas et al., 2003; Heinrich-Weltzien et al., 2005; Stookey, 2005; Pretty, 2006; Pretty and Ellwood, 2007], but it is subject to confounding effects of saliva [Amaechi and Higham, 2002], drying time [Pretty et al., 2004], angulation [Buchalla et al., 2002; Ando et al., 2004a] and stain [Shi et al., 2001] among others factors [Heinrich-Weltzien et al., 2005]. Additionally, although QLF parameters (lesion depth expressed as $\Delta \mathrm{F}$ and lesion volume expressed as $\Delta \mathrm{Q}$ ) have been shown to have a higher sensitivity when compared to other methods for detection of early caries lesions [Ferreira Zandoná et al., 2003] because the QLF analysis software detects any change in pixel values as demineralization, their specificity is greatly compromised. In a previous study, allowing the examiner to determine from the QLF image the presence or absence of a lesion yielded the best combination of sensitivity and specificity values for the detection of occlusal and smooth surface caries on deciduous teeth [Ferreira Zandoná et al., 2003]. Therefore, if QLF can be used to supplement clinical caries detection, such as by using ICDAS criteria, it may be possible to have clinically meaningful assessments by providing measurements over and above the noise of arrested initial and subclinical lesions [Pitts, 2004]. This article describes a methodology being used in a 4-year longitudinal study where a combination of ICDAS and QLF is being used to monitor lesion progression.

\section{Subjects and Methods}

Children $(\mathrm{n}=569)$ from kindergarten to 9th grade public schools in the area of Aguas Buenas, Puerto Rico, were recruited and consented in January 2007, as approved by the institutional review board committees of Indiana University and the University of Puerto Rico (IU IRB No. 0608-15 and UPR IRB No. A1340107). The sample size was chosen based on preliminary data in order to have a sufficient number of clinically significant lesions at the end of the 4-year study. For inclusion in the study, children had to be between 5 and 13 years of age, have at least 1 permanent molar with at least 1 unrestored surface, have no medical problem that contraindicated participation, and allow examination of the oral cavity, including radiographs and digital photographs.

The children received an oral soft tissue examination and an examination by a single calibrated examiner using the ICDAS criteria followed by a QLF examination using modified ICDAS criteria (QLF-I) at baseline, 8 months and 12 months (table 1). Full mouth examinations were conducted using the ICDAS criteria following the committee guidelines [Ismail et al., 2007], but only the occlusal and buccal surfaces of all permanent molars as well as the lingual surface of upper molars were examined and imaged by QLF (QLF Pro; Inspektor Research Systems, Amsterdam, The Netherlands). QLF examinations were conducted in a controlled, darkened environment immediately after the same examiner had completed the ICDAS examination. The ICDAS data, collected in tablet PCs using a custom-made software (Optiform, Indianapolis, Ind., USA), were transferred from the ICDAS form to the QLF-I form. In this manner, the examiner was able to determine if the lesion was scored using the ICDAS criteria prior to determining the QLF-I score. This 'bias' was tolerable to allow a real-life combination of the 2 methods, which was expected to allow allocating a higher specificity to QLF without decreasing its sensitivity. Repeat examinations were conducted in $10 \%$ of the children. Prior to the examinations, the teeth were brushed and flossed by study personnel. 
Table 2. Association between ICDAS and QLF-I ${ }^{1}$

\begin{tabular}{|c|c|c|c|c|c|c|c|c|}
\hline & \multirow[t]{2}{*}{ QLF-I } & \multicolumn{7}{|c|}{ ICDAS } \\
\hline & & 0 & 1 & 2 & 3 & 4 & 5 & 6 \\
\hline Occlusal, buccal pit and & 0 & 5,998 & 103 & 15 & 0 & 0 & 0 & 0 \\
\hline \multirow[t]{5}{*}{ lingual groove surfaces } & 1 & 1,374 & 913 & 93 & 0 & 1 & 0 & 0 \\
\hline & 2 & 306 & 954 & 1,160 & 4 & 8 & 0 & 1 \\
\hline & 3 & 2 & 0 & 3 & 305 & 0 & 0 & 0 \\
\hline & 4 & 1 & 15 & 114 & 54 & 204 & 0 & 1 \\
\hline & 5 & 0 & 0 & 0 & 2 & 0 & 223 & 138 \\
\hline \multirow[t]{6}{*}{ Smooth surfaces } & 0 & 8,365 & 309 & 67 & 3 & 0 & 0 & 0 \\
\hline & 1 & 597 & 786 & 203 & 0 & 0 & 0 & 0 \\
\hline & 2 & 105 & 128 & 708 & 2 & 0 & 0 & 0 \\
\hline & 3 & 0 & 1 & 0 & 35 & 0 & 0 & 0 \\
\hline & 4 & 2 & 0 & 0 & 0 & 0 & 0 & 0 \\
\hline & 5 & 0 & 0 & 1 & 1 & 0 & 55 & 43 \\
\hline \multirow[t]{6}{*}{ Combined surfaces } & 0 & 14,363 & 412 & 82 & 3 & 0 & 0 & 0 \\
\hline & 1 & 1,971 & 1,699 & 296 & 0 & 1 & 0 & 0 \\
\hline & 2 & 411 & 1,082 & 1,868 & 6 & 8 & 0 & 1 \\
\hline & 3 & 2 & 1 & 3 & 340 & 0 & 0 & 0 \\
\hline & 4 & 3 & 15 & 114 & 54 & 204 & 0 & 1 \\
\hline & 5 & 0 & 0 & 1 & 3 & 0 & 278 & 181 \\
\hline
\end{tabular}

${ }^{1}$ ICDAS scores 5 and 6 were collapsed on QLF-I scores.

Repeatability of the ICDAS and QLF-I examinations was assessed using weighted kappa statistics. The association between ICDAS and QLF-I scores was evaluated using two-way contingency tables and Kendall's tau-b correlation coefficients.

\section{Results}

A total of 569 children completed the baseline examination, 484 children completed the 8 -month examination and 460 children completed the 12-month examination. The average age at baseline was 9.7 (SD 2.2) years. The vast majority of the children were Hispanic (91\%) and $51 \%$ were males. On average, the DMFT score was 6.0 (SD 5.8) at baseline and 6.4 (SD 6.3) at 12 months. At baseline, $9.5 \%$ of the children had at least 1 sealant and $63 \%$ of the children had at least 1 filling. The average number of baseline surfaces with an ICDAS score of at least 1 was 14.5 (SD 11.2); 6.7 (SD 6.6) surfaces had a score of at least 3 , and 5.8 (SD 6.2) had a score of at least 5.

Combining the repeat examinations performed at the baseline and 12-month exams, the intraexaminer repeatability was similar for the ICDAS and QLF-I examinations (results are presented only for surfaces evaluated by both methods). The weighted kappa for the ICDAS was $0.78,0.70$ and 0.76 for occlusal/buccal pit/lingual groove surfaces, smooth surfaces and combined surfaces, respectively. The weighted kappa for the QLF-I was $0.79,0.72$ and 0.78 for occlusal/buccal pit/lingual groove surfaces, smooth surfaces and combined surfaces, respectively.

To evaluate the relationship between the ICDAS and QLF-I examinations, data from the baseline, 8-month and 12-month examinations were combined. The correlations between ICDAS and QLF-I examinations were $0.79,0.74$ and 0.77 for occlusal/buccal pit/lingual groove surfaces, smooth surfaces and combined surfaces, respectively (table 2). QLF-I examinations scored more lesions as 1 and 2 than the ICDAS. Additionally, more codes 4 were scored during the QLF-I examination than during the ICDAS examinations on occlusal surfaces.

Both examinations were able to detect changes in lesions from baseline to 12 months (table 3). Using the ICDAS, $21 \%$ of the occlusal/buccal pit/lingual groove surfaces and $11 \%$ of the smooth surfaces showed progression of the lesion scores between the baseline and 12-month examinations. A greater percentage of surfaces were determined to have increased lesion scores using the QLF-I, where $29 \%$ of the occlusal/buccal pit/lingual 
Table 3. Score transitions between baseline and 12-month examination

a ICDAS score transitions

\begin{tabular}{|c|c|c|c|c|c|c|c|c|}
\hline & \multirow[t]{2}{*}{ Baseline } & \multicolumn{7}{|c|}{12 months } \\
\hline & & 0 & 1 & 2 & 3 & 4 & 5 & 6 \\
\hline Occlusal, buccal pit and & 0 & 1,850 & 250 & 70 & 11 & 4 & 6 & 0 \\
\hline \multirow[t]{6}{*}{ lingual groove surfaces } & 1 & 82 & 232 & 137 & 24 & 9 & 3 & 0 \\
\hline & 2 & 17 & 43 & 171 & 56 & 32 & 17 & 2 \\
\hline & 3 & 0 & 1 & 11 & 28 & 18 & 24 & 2 \\
\hline & 4 & 0 & 2 & 0 & 0 & 26 & 5 & 1 \\
\hline & 5 & 0 & 0 & 1 & 1 & 1 & 28 & 10 \\
\hline & 6 & 0 & 0 & 1 & 0 & 0 & 0 & 23 \\
\hline \multirow[t]{7}{*}{ Smooth surfaces } & 0 & 2,318 & 179 & 73 & 1 & 0 & 4 & 2 \\
\hline & 1 & 155 & 132 & 72 & 0 & 0 & 2 & 0 \\
\hline & 2 & 28 & 42 & 162 & 6 & 0 & 3 & 0 \\
\hline & 3 & 2 & 0 & 5 & 1 & 0 & 1 & 0 \\
\hline & 4 & 0 & 0 & 0 & 0 & 0 & 0 & 0 \\
\hline & 5 & 2 & 0 & 0 & 0 & 0 & 9 & 2 \\
\hline & 6 & 0 & 0 & 0 & 0 & 0 & 0 & 6 \\
\hline \multirow[t]{7}{*}{ Combined surfaces } & 0 & 4,168 & 429 & 143 & 12 & 4 & 10 & 2 \\
\hline & 1 & 237 & 364 & 209 & 24 & 9 & 5 & 0 \\
\hline & 2 & 45 & 85 & 333 & 62 & 32 & 20 & 2 \\
\hline & 3 & 2 & 1 & 16 & 29 & 18 & 25 & 2 \\
\hline & 4 & 0 & 2 & 0 & 0 & 26 & 5 & 1 \\
\hline & 5 & 2 & 0 & 1 & 1 & 1 & 37 & 12 \\
\hline & 6 & 0 & 0 & 1 & 0 & 0 & 0 & 29 \\
\hline
\end{tabular}

b QLF-I score transitions

\begin{tabular}{llrrrrrr}
\hline & Baseline & \multicolumn{2}{l}{ 12 months } & & & & \\
\cline { 2 - 7 } & & 0 & 1 & 2 & 3 & 4 & 5 \\
\hline Occlusal, buccal pit and & 0 & 1,391 & 344 & 102 & 6 & 2 & 3 \\
lingual groove surfaces & 1 & 65 & 264 & 269 & 14 & 8 & 2 \\
& 2 & 9 & 36 & 331 & 63 & 55 & 15 \\
& 3 & 0 & 0 & 9 & 18 & 19 & 22 \\
& 4 & 1 & 1 & 6 & 4 & 57 & 19 \\
Smooth surfaces & 5 & 0 & 1 & 1 & 1 & 0 & 61 \\
& 0 & 2,220 & 205 & 49 & 1 & 1 & 8 \\
& 1 & 136 & 247 & 60 & 0 & 0 & 2 \\
& 2 & 10 & 59 & 174 & 6 & 0 & 2 \\
& 3 & 1 & 1 & 4 & 1 & 0 & 1 \\
& 4 & 0 & 0 & 0 & 0 & 0 & 0 \\
& 5 & 1 & 0 & 1 & 0 & 0 & 17 \\
& 0 & 3,611 & 549 & 151 & 7 & 3 & 11 \\
& 1 & 201 & 511 & 329 & 14 & 8 & 4 \\
& 2 & 19 & 95 & 505 & 69 & 55 & 17 \\
& 3 & 1 & 1 & 13 & 19 & 19 & 23 \\
& 4 & 1 & 1 & 6 & 4 & 57 & 19 \\
& 5 & 1 & 1 & 2 & 1 & 0 & 78 \\
\hline
\end{tabular}


groove surfaces and $10 \%$ of the smooth surfaces showed progression of the lesion scores. Interestingly, smooth surface lesions that were scored 2 using either method at baseline rarely progressed by the 12 -month examination and often had a reduced score at 12 months.

\section{Discussion}

It has been reported that dentists are diagnosing young patients and adults with occlusal lesions at the noncavitated level more frequently [Kühnisch et al., 2001] and the ICDAS is particularly useful in clinical investigations, adding lesions that would not be recorded in the WHO system [Kühnisch et al., 2008]. Although the sensitivity of the visual method using the ICDAS criteria is higher (0.59-0.73) than traditionally reported [Jablonski-Momeni et al., 2008; Rodrigues et al., 2008], it is still lower than that of some of the technology-based methods. Nevertheless, to date, none of these technology-based methods can be used as a stand-alone approach. They should be an adjunct to clinical decision-making to aid in early caries diagnosis since detection is only part of the diagnostic process necessary to properly assess a patient's caries risk status [Zandoná and Zero, 2006]. QLF has shown great promise as an early caries detection method, but it is weighed down by several confounding factors such as stain [Shi et al., 2001], saliva [Amaechi and Higham, 2002], dehydration [Pretty et al., 2004] and angulation [Ando et al., 2004a], which can limit its applications. The combination of this method with the ICDAS is a great fit to allow the best characteristics of both methods to come forward: the high sensitivity of QLF and the high specificity of the visual method. This ongoing longitudinal study has combined ICDAS examinations with QLF to monitor lesion progression.

Both methods, the ICDAS and QLF, were able to follow the increase in average DMFT scores from baseline to 12 months. Even though QLF in our study has been used as a visual aid, and the analysis function has not been used, the examination aided by QLF detected more lesions, mainly of scores 1 and 2, than the ICDAS, similar to the findings by Kühnisch et al. [2008], where analyses of QLF images detected more lesions than visual examination. It is of interest to observe that QLF detected more scores deemed as 4 using the QLF-I criteria (almost 50\% more) than the ICDAS on occlusal surfaces, which is of clinical significance as these lesions are expected to be in the inner half of the dentin, compared to lesions of ICDAS scores 2, which would be expected to be in the in- ner enamel or outer dentin. Although the QLF-I criteria are not in vitro-validated criteria, the data shown here indicate an alignment with the ICDAS criteria. Further analyses of the images and follow-up of the progression of the lesions should confirm this trend.

Nevertheless, detecting early lesions is of little significance if these lesions are not active and do not progress. In our population, during this 12 -month period more lesions on occlusal surfaces were transitioning to a more severe score (34\% of the lesions scored as ICDAS 1 or 2 at baseline) than transitioning to a less severe score ( $17 \%$ of the lesions scored as ICDAS 1 or 2 at baseline). This result is similar to those found by Rugarabamu et al. [2002], who reported a progression of $30-47.9 \%$ of occlusal lesions at 1 year, even though that population had a lower caries prevalence than the population in our study. For lesions on smooth surfaces, the opposite was true (14\% transitioning to a more severe score vs. $37 \%$ transitioning to a less severe score). This trend was similar for the lesions detected by QLF-I, with 38\% of lesions on occlusal surfaces scoring 1 or 2 at baseline transitioning to a more severe score at 12 months and $10 \%$ transitioning to a less severe score. Again, lesions on smooth surfaces had the opposite trend: $10 \%$ transitioned to a more severe score, while $30 \%$ transitioned to a less severe score.

In conclusion, the combined methodology has the clinical potential to allow earlier detection of lesions, which on occlusal surfaces are likely to progress.

\section{Acknowledgments}

This study was supported by grant No. NIH-RO1 DEO1789004 from the National Institute of Dental and Craniofacial Research, NIH, Bethesda, Md., USA. The authors would like to thank the following individuals for their assistance: Ms. Sharon Gwinn, Ms. Myrna Hernandez, Ms. Melissa Mau, Ms. Mildred Riviera, Ms. Jenniffer Tran, Drs. Evaristo Delgado, Hafsteinn Eggertsson and Pedro Hernandez, and the supporting staff at Indiana Oral Health Research Institute and the University of Puerto Rico.

References Amaechi BT, Higham SM: Quantitative lightinduced fluorescence: a potential tool for general dental assessment. J Biomed Opt 2002;7:7-13.

Amaechi BT, Podoleanu A, Higham SM, Jackson DA: Correlation of quantitative lightinduced fluorescence and optical coherence tomography applied for detection and quantification of early dental caries. J Biomed Opt 2003;8:642-647. 
Ando M, Eckert GJ, Stookey GK, Zero DT: Effect of imaging geometry on evaluating natural white-spot lesions using quantitative lightinduced fluorescence. Caries Res 2004a;38: 39-44.

-Ando M, Gonzalez-Cabezas C, Isaacs RL, Eckert GJ, Stookey GK: Evaluation of several techniques for the detection of secondary caries adjacent to amalgam restorations. Caries Res 2004b;38:350-356.

-Ando M, Hall AF, Eckert GJ, Schemehorn BR, Analoui M, Stookey GK: Relative ability of laser fluorescence techniques to quantitate early mineral loss in vitro. Caries Res 1997; 31:125-131.

-Ando M, van der Veen MH, Schemehorn BR, Stookey GK: Comparative study to quantify demineralized enamel in deciduous and permanent teeth using laser- and light-induced fluorescence techniques. Caries Res 2001;35: 464-470.

-Angmar-Mansson B, ten Bosch JJ: Quantitative light-induced fluorescence (QLF): a method for assessment of incipient caries lesions. Dentomaxillofac Radiol 2001;30:298-307.

Buchalla W, Lennon AM, van der Veen MH, Stookey GK: Optimal camera and illumination angulations for detection of interproximal caries using quantitative light-induced fluorescence. Caries Res 2002;36:320-326.

Burt BA, Kolker JL, Sandretto AM, Yuan Y, Sohn W, Ismail AI: Dietary patterns related to caries in a low-income adult population. Caries Res 2006;40:473-480.

Cook SL, Martinez-Mier EA, Dean JA, Weddell JA, Sanders BJ, Eggertsson H, Ofner S, Yoder $\mathrm{K}$ : Dental caries experience and association to risk indicators of remote rural populations. Int J Paediatr Dent 2008;18:275-283.

de Josselin de Jong E, Sundström F, Westerling $\mathrm{H}$, Tranaeus S, ten Bosch JJ, Angmar-Mansson B: A new method for in vivo quantification of changes in initial enamel caries with laser fluorescence. Caries Res 1995;29:2-7.

-Ekstrand KR, Martignon S, Ricketts DJ, Qvist V: Detection and activity assessment of primary coronal caries lesions: a methodologic study. Oper Dent 2007;32:225-235.
Ferreira Zandoná AG, Stookey GK, Eggertsson H, Wefel J, Katz B, Ofner S, Eckert G: Clinical validation study of QLF at Indiana; in Stookey GK (ed): Proceedings of the 6th Annual Indiana Conference. Indiana University School of Dentistry, 2003, vol 6, pp 237-252.

Finlayson TL, Siefert K, Ismail AI, Sohn W: Psychosocial factors and early childhood caries among low-income African-American children in Detroit. Community Dent Oral Epidemiol 2007;35:439-448.

Gonzalez-Cabezas C, Fontana M, Gomes-Moosbauer D, Stookey GK: Early detection of secondary caries using quantitative light-induced fluorescence. Oper Dent 2003;28: 415-422.

Heinrich-Weltzien R, Kühnisch J, Ifland S, Tranaeus S, Angmar-Mansson B, Stösser L: Detection of initial caries lesions on smooth surfaces by quantitative light-induced fluorescence and visual examination: an in vivo comparison. Eur J Oral Sci 2005;113:494498.

-Ismail AI, Sohn W, Tellez M, Amaya A, Sen A, Hasson H, Pitts NB: The International Caries Detection and Assessment System (ICDAS): an integrated system for measuring dental caries. Community Dent Oral Epidemiol 2007;35:170-178.

Ismail AI, Sohn W, Tellez M, Willem JM, Betz J, Lepkowski J: Risk indicators for dental caries using the International Caries Detection and Assessment System (ICDAS). Community Dent Oral Epidemiol 2008;36:55-68.

Jablonski-Momeni A, Stachniss V, Ricketts DN, Heinzel-Gutenbrunner M, Pieper K: Reproducibility and accuracy of the ICDAS-II for detection of occlusal caries in vitro. Caries Res 2008;42:79-87.

Kühnisch J, Berger S, Goddon I, Senkel H, Pitts N, Heinrich-Weltzien R: Occlusal caries detection in permanent molars according to WHO basic methods, ICDAS II and laser fluorescence measurements. Community Dent Oral Epidemiol 2008;36:475-484.

Kühnisch J, Heinrich-Weltzien R, Senkel H, Sonju Clasen AB, Stösser L: Dental health and caries topography in 8-year-old German and immigrant children. Eur J Paediatr Dent 2001;2:191-196.

Pitts N: 'ICDAS': an international system for caries detection and assessment being developed to facilitate caries epidemiology, research and appropriate clinical management. Community Dent Health 2004;21:193-198.
Pretty IA: Caries detection and diagnosis: novel technologies. J Dent 2006;34:727-739.

Pretty IA, Edgar WM, Higham SM: The effect of dehydration on quantitative light-induced fluorescence analysis of early enamel demineralization. J Oral Rehabil 2004;31:179-184.

Pretty IA, Ellwood RP: Comparison of paired visual assessment and software analyses of changes in caries status over 6 months from fluorescence images. Caries Res 2007;41: 115-120.

-Rodrigues JA, Hug I, Diniz MB, Lussi A: Performance of fluorescence methods, radiographic examination and ICDAS II on occlusal surfaces in vitro. Caries Res 2008;42: 297-304.

Rugarabamu PG, Poulsen S, Masalu JR: A longitudinal study of occlusal caries among schoolchildren in Dar es Salaam, Tanzania. Community Dent Oral Epidemiol 2002;30: 47-51.

Shi XQ, Tranaeus S, Angmar-Mansson B: Comparison of QLF and DIAGNOdent for quantification of smooth surface caries. Caries Res 2001;35:21-26.

Sohn W, Ismail A, Amaya A, Lepkowski J: Determinants of dental care visits among lowincome African-American children. J Am Dent Assoc 2007;138:309-318, quiz 395-396.

Stookey GK: Quantitative light fluorescence: a technology for early monitoring of the caries process. Dent Clin North Am 2005;49:753770 .

-Tranaeus S, Al-Khateeb S, Björkman S, Twetman S, Angmar-Mansson B: Application of quantitative light-induced fluorescence to monitor incipient lesions in caries-active children: a comparative study of remineralisation by fluoride varnish and professional cleaning. Eur J Oral Sci 2001;109:71-75.

Varma S, Banerjee A, Bartlett D: An in vivo investigation of associations between saliva properties, caries prevalence and potential lesion activity in an adult UK population. J Dent 2008;36:294-299.

Zandoná AF, Zero DT: Diagnostic tools for early caries detection. J Am Dent Assoc 2006;137: 1675-1684, quiz 1730, erratum 2007;138: 298. 